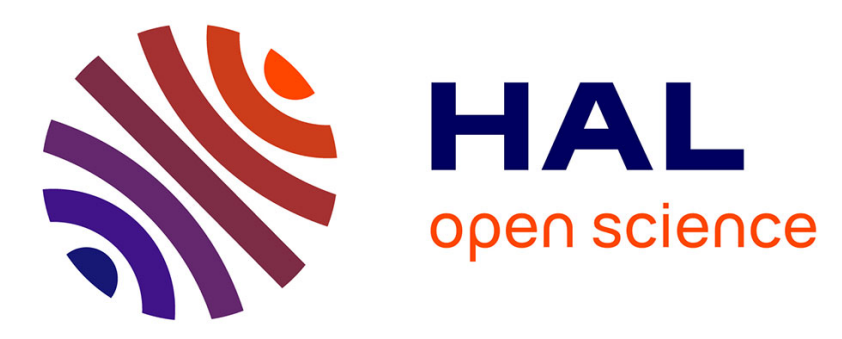

\title{
Drivers' phone use at red traffic lights: A roadside observation study comparing calls and visual-manual interactions
}

\author{
Véronique Huth, Yann Sanchez, Corinne Brusque
}

\section{To cite this version:}

Véronique Huth, Yann Sanchez, Corinne Brusque. Drivers' phone use at red traffic lights: A roadside observation study comparing calls and visual-manual interactions. Accident Analysis \& Prevention, 2015, 74, pp.42-48. 10.1016/j.aap.2014.10.008 . hal-01807488

\section{HAL Id: hal-01807488 \\ https://hal.science/hal-01807488}

Submitted on 4 Jun 2018

HAL is a multi-disciplinary open access archive for the deposit and dissemination of scientific research documents, whether they are published or not. The documents may come from teaching and research institutions in France or abroad, or from public or private research centers.
L'archive ouverte pluridisciplinaire $\mathbf{H A L}$, est destinée au dépôt et à la diffusion de documents scientifiques de niveau recherche, publiés ou non, émanant des établissements d'enseignement et de recherche français ou étrangers, des laboratoires publics ou privés. 
1 Drivers' phone use at red traffic lights: a roadside observation study comparing calls and visual-manual interactions

3 Véronique Huth ${ }^{1}$, Yann Sanchez ${ }^{1}$, Corinne Brusque ${ }^{1}$

${ }^{1}$ Université de Lyon, IFSTTAR (TS2/LESCOT)

25, avenue François Mitterrand

69675 Bron, France

Email: veronique.huth@ifsttar.fr (corresponding author)

Tel: 0033-652486886

Abstract

Phone use while driving has become one of the priority issues in road safety, given that it may lead to a decrease in situation awareness and deteriorated driving performance. It has been suggested that drivers can regulate their exposure to secondary tasks and seek for compatibility of phone use and driving. Phone use strategies include the choice of driving situations with low demands and interruptions of the interaction when the context changes. Traffic light situations at urban intersections imply both a temptation to use the phone while waiting at the red traffic light and a potential threat due to the incompatibility of phone use and driving when the traffic light turns green. These two situations were targeted in a roadside observation study, with the aim to investigate the existence of a phone use strategy at the red traffic light and to test its effectiveness. $\mathrm{N}=124$ phone users and a corresponding control group of non-users were observed. Strategic phone use behaviour was detected for visualmanual interactions, which are more likely to be initiated at the red traffic light and tend to be stopped before the vehicle moves off, while calls are less likely to be limited to the red traffic light situation. As an indicator of impaired situation awareness, delayed start was associated to phone use and in particular to visual-manual interactions, whether phone use was interrupted before moving off or not. Traffic light situations do not seem to allow effective 
1 application of phone use strategies, although drivers attempt to do so for the most demanding

2 phone use mode. The underlying factors of phone use need to be studied so as to reduce the temptation of phone use and facilitate exposure regulation strategies.

\section{Keywords}

Mobile phone, distraction, observation, traffic light, use strategy, situation awareness, visual-

7 manual interaction

\section{Introduction}

Communication is a central element of the digital age, and phone use has evolved into a habit for many people. The statistics of the International Telecommunication Union show that mobile phone subscriptions have dramatically increased over the last decade (ITU, 2014). Inevitably, phone use is also taking place in road traffic and driver distraction by mobile phones has become one of the priority issues in road safety (WHO, 2011). In a survey conducted in 2012 with a representative sample of the French driver population, $23 \%$ of the respondents indicated to use the phone with a hands-free kit while driving and $11 \%$ admitted to use the phone hand-held. $13 \%$ of the respondents said to read text (messages, mails or websites) and $11 \%$ to write text while driving (ONISR, 2013). Accident analyses conducted in 2010 revealed that close to $10 \%$ of injury crashes in France could be attributed to mobile phone use while driving (OECD/ITF, 2014). On an international level, reviews of the effects of phone use on driving performance keep raising concerns on the cognitive, visual and manual distraction provoked by calls or other interactions with the phone and highlight the corresponding increase in crash risk (McCartt et al., 2006; Brace et al., 2007; Kircher et al., 2011; Bruyas, 2013). Given that they provoke interruptions of drivers' visual sampling of the 
1 driving environment, distracting activities with high visual demand are associated to higher

2 crash risk than those that only imply cognitive distraction (Young \& Salmon, 2012).

3 Simulated as well as naturalistic driving studies revealed a relationship between crashes and

4 glances inside the vehicle that lasted more than 1.6 or 2 seconds respectively (Horrey \&

5 Wickens, 2007; Klauer et al., 2006). Related to phone use, the greatest increases in the odds

6 for drivers to be involved in safety-critical events have been found for text messaging,

7 followed by dialling a number and reaching for a mobile device (Hickman et al., 2010; Olson

8 et al., 2009).

9 While drivers used a mobile phone, failures to perceive and process traffic signs and hazards that are present on the road have been found (McKnight \& McKnight, 1993) and longer reaction times to traffic signals and events have been observed (Strayer et al., 2003; Charlton, 2009). Hence, phone use can lead to a general decrease in situation awareness, as defined by Endsley (1988), and to violations of traffic rules (Caird et al., 2008). These effects are amplified if the drivers' use of the mobile phone implies a diversion of the gaze towards the mobile phone. For example, while texting drivers make more in-vehicle glances and reduce their visual attention to the environment, which has proven to be associated to impaired lateral vehicle control (Hosking et al., 2009; Drews et al., 2009). In addition to their decreased awareness of changing events in the road scene, drivers might not concentrate on the traffic situation for long enough between their off-road glances so as to get back 'in-the-loop' (Senders et al., 1967 cited in Young \& Salmon, 2012). As a consequence, drivers suffer from delayed detection of relevant information or complete hazard identification failures. Thus, a major issue of concern lies in the alteration of visual information uptake when using the phone.

The latest generations of mobile phones feature a wide range of use options, with a growing number of mobile phone applications that require visual-manual interactions. Smartphones are 
1 not only increasingly popular; they also tend to become an essential device in everyday life and imply strong temptations for visual-manual interactions. Their use can become an automated habit, driven by internal factors, such as the need to constantly stay informed and in touch with the social network, or by external triggers, i.e. a sound emitted by the phone. These triggers can appear at any time and in any situation, and consequently also while driving (Bayer \& Campbell, 2012).

In their deciding-to-be-distracted approach, Lerner and Boyd (2005) argue that the risk of secondary tasks while driving is not only determined by the distractive potential of the activity itself but also by the driver's exposure to the task, and that drivers can influence their risk by actively regulating exposure to secondary tasks. This regulation is based on higherlevel decisions, which depend on motivational and cognitive factors (Summala, 1997). It can either be achieved by exposure limitation or by seeking for compatibility of the secondary task and driving, i.e. by choosing traffic situations in which the demands of the driving tasks leave enough attentional resources for the phone use (Huth \& Brusque, 2013). In a simulator study, Schömig et al. (2011) observed that drivers took the deliberate decision to engage in the secondary task based on an anticipation of the development of situational demands and the judgement of their compatibility with the execution of a concurrent task. Specifically, Stutts et the driving task can vary dramatically, since the demands of driving can be relatively low or extremely high, according to the driving situation and the manoeuvre the driver is carrying out. The traffic context is dynamic and can evolve quickly, and drivers will have to apply phone use strategies that are adapted to these changing conditions. 
1 regard, the possibility to interrupt the phone interaction plays a crucial role (Huth \& Brusque,

2 2013). Interruptions of secondary tasks in order to execute controls of the primary task have

3 been described as an interaction strategy with in-vehicle devices (Rauch et al., 2008).

4 However, phone interactions might not always be easily dividable into several chunks that

5 allow the driver to pay attention to the traffic situations at regular and appropriate intervals.

6 Calls might be difficult to interrupt quickly, given that the conversation partner is not

7 witnessing the traffic situation, whereas interruptions of visual-manual phone use can lead to

8 interaction errors, which can also extend the interaction beyond the drivers' intentions.

9 In this regard, intersections represent a particularly interesting location due to the dynamic driving context that can considerably evolve within short periods of time (Sandin, 2009).

Driving through an intersection implies different phases that are associated to driving tasks of different difficulty levels (Cooper et al., 2003). Furthermore, intersections are locations where the paths of several types of road users cross, which can lead to conflicts with potentially severe outcomes (Habibovic \& Davidson, 2012). At the same time, intersections controlled by traffic lights can induce drivers to engage in phone use while safely stopped at a red traffic light. In this situation, drivers may take the chance to use the phone out of necessity or boredom, including highly demanding phone use modes such as texting or checking emails. However, once the traffic light turns green, the driver enters a potentially complex situation with high attentional demands. Negative consequences of phone use could appear in this situation if the phone interaction was not interrupted when starting to move the vehicle or if the driver is still cognitively distracted by the recent phone use.

In sum, traffic light situations at urban intersections imply both a temptation to use the phone at the red traffic light and a potential threat due to the incompatibility of phone use and the driving task when the traffic signal turns green and the vehicle moves off. The aim of this 
1 study was to observe phone use at urban intersections during these two situations and to

2 address the following two research questions:

3 1. The first research question focussed on the existence of a phone use strategy at the red

4 traffic light. The aim was to determine if drivers took the chance to use their phone when

5 waiting at a red traffic light by observing the initiation of phone use in this situation.

6 2. The second research question concerned the effectiveness of the phone use strategy at red

7 traffic lights. It was addressed in two parts. Firstly, the cessation of phone use at traffic lights

8 was observed in order to examine if drivers limit the phone use to the safe stopping situation.

9 Secondly, the relationship between phone use and delayed starts when the traffic signal turns 10 green was tested.

11 Given that phone calls and visual-manual interactions affect driving to a different extent, the existence and effectiveness of a phone use strategy were tested for differences between these two phone use modes.

\section{Method}

\subsection{Observations}

The method applied in this study is based on traffic observation techniques, which have previously been used in studies related to the prevalence of distracting secondary activities while driving (Sullman, 2012), and in particular regarding mobile phone use (Walker et al., 2006; Pickrell \& Ye, 2010). In these studies, trained observers collect information on driver characteristics and predefined target behaviours in the setting where they naturally occur. Behaviour is measured directly, avoiding possible biases of self-reports, and the noninvasiveness of this research method makes for high construct and face validity (Eby, 2011). 
1 According to the research questions, the present study collected information on drivers who

2 used their mobile phone while waiting at a red traffic light. With the aim to compare driver

3 behaviour of mobile phone users and non-users, a control group of non-users was created in

4 parallel.

5 Three urban intersections that are controlled by traffic lights were selected in Lyon, France.

6 At each intersection, the traffic light approach zone with best visibility for the observers and

7 most convenient traffic flow was chosen. The observation area comprised the queue of the

8 first four vehicles stopped at the red traffic light, on two parallel lanes. Drivers who passed

9 while the traffic light was green were not included into the sample. The observations were carried out on-site by the observers rather than from video recordings, accounting for privacy concerns as well as for the difficulty to detect and precisely characterize phone uses from outside the vehicle. The observers were placed on the sidewalk opposite the observed lanes, in order to be less visible to the observed drivers and to have good visibility on the observation zone, including the traffic light signal. They were not assigned a fix point, in order to be able to adapt their position depending on the driver they were observing.

\subsection{Procedure}

In order to specify the observation variables and develop a corresponding observation grid, ad hoc observations were been carried out on the three selected observation sites. During this pre-test phase, two observers wrote down any noticeable detail that could be related to the research questions. Visible phone use modes and an observable indicator for the drivers' situation awareness were defined. During this process, the observers made sure that the variables were accurately observable from the roadside. At the same time, they were sensitised and trained for the observation of phone use in the chosen context. 
1 Once the variables had been defined and integrated into a standardized observation grid,

2 further training sessions were carried out until the observers converged towards common

3 criteria for each observed variable. After about one hour at each observation site the observers

4 had reached an interobserver reliability that was greater than $85 \%$ (Eby, 2011). During the ad-

5 hoc observations and training sessions, the observers gained experience specifically related to

6 the detection of different phone use modes, with the aim to increase sensitivity, i.e. their

7 ability to track down phone use, and specificity, i.e. their ability to discriminate phone

8 interactions from other secondary activities inside the car, such as the interaction with in-

$9 \quad$ vehicle controls.

Standardized observations then took place in June and July 2013 on weekdays between 4 pm and $7 \mathrm{pm}$, ensuring a steady traffic flow that corresponded to evening rush hour traffic. Observation sessions lasted 50 minutes, followed by a break of ten minutes. Data was collected during three sessions at each observation site, resulting in a total of nine sessions and 7.5 hours of observation. Weather conditions were good during all observation sessions. Data was collected paper-and-pencil, using the standardized observation grid that had been specifically created based on the ad hoc observations.

The instruction given to the observers was to fill in the observation grid for each driver using a mobile phone while waiting at the red light. Exhaustiveness of this data collection could not be guaranteed, but the coding grid was designed so as to minimize the coding time and to maximize the number of observed phone users. For each driver who was observed using a mobile phone while waiting at the red light (target group), a driver who was not using the phone was observed (control group). The non-user was selected randomly, with the only requirement to occupy a similar position in the queue and to be observed in a narrow time frame after the user. These requirements were set to ensure that the traffic situation of the non-user resembled the one of the corresponding user. During each observation session, one 
1 observer registered the target group and the other observer took over data collection for the control group. The roles of the observers were counterbalanced among sessions so as to avoid observation artefacts.

\subsection{Measures}

All measures taken in this study were observation variables, which were included in the standardized grid that had been developed on the basis of the ad-hoc observations. In order to facilitate data collection, the variables were aligned in a row from the left to the right of the observation grid, and the observers simply had to tick boxes next to the variable categories that applied to the driver they were observing.

The first variable registered in the observation grid was phone use, distinguishing between users and non-users. All observed drivers were then assigned a vehicle type, gender and estimated age category. The vehicle type distinguished between passenger cars and minivans. Drivers of other vehicles were not included into the sample. Three age categories were coded according to previous studies on mobile phone use (e.g., Sullman, 2012; Young et al., 2010). The first category included those drivers who appeared to be under thirty years of age, the second one those between thirty and fifty, and the third category comprised those drivers who were judged as being over fifty.

The observed phone uses were assigned to three categories, which were based on the visible phone use modes collected in the pre-tests and which follow the categorization made in the NTHSA driver electronic device use observation protocol (Pickrell \& Ye, 2010). Hands-free calls cover conversations held with the help of any hands-free device such as an integrated car-kit, a Bluetooth ear set or headphones; hand-held calls include conversations with the phone held to the ear or with the phone held in hand using the speaker of the phone; and 
1 visual-manual interactions are all phone uses except calls, which involve visual attention

2 towards the phone and/or its manipulation and which can range from checking the screen of

3 the phone to inserting a text. Drivers wearing headphones without speaking were not

4 registered as phone users.

5 The observed phone use was furthermore characterized by its beginning with respect to the

6 red traffic light situation, distinguishing between phone uses that were initiated while waiting

7 at the red traffic light and those that were already ongoing when the driver entered the red

8 traffic light situation. Similarly, the drivers' phone use behaviour after the end of the traffic

9 light situation was coded, differentiating phone uses that were stopped when the traffic light turned green from those that were continued.

As an observable indicator for the drivers' situation awareness the drivers' response to the change of the traffic light signal was assessed, indicating if the drivers moved off as soon as it was correct and safe to do so. If the observer judged that it took the driver longer than other drivers to react to the green traffic light, this behaviour was coded as a delayed start. This variable was registered for users and non-users.

\subsection{Data analysis}

Data was cross-classified in $\mathrm{RxC}$ contingency tables and compared using chi-square tests of independence with a significance level of $p<.05$. Chi-squared tests were calculated to test for differences between phone use modes and between the behaviour of users and non-users. For the tests on differences between phone use modes, counts of hands-free and hand-held calls were collapsed into one variable (calls) and compared to the counts of visual-manual interactions, given that phone calls and visual-manual interactions affect driving to a different extent. Phi was calculated to measure effect sizes. 


\section{Results}

2

3

4

\subsection{Observed phone users}

In total, 124 drivers who used the phone while waiting at the traffic light were observed across the three observation sites. Correspondingly, data on 124 non-users were collected. $54.0 \%$ of the observed phone uses were calls, consisting of 44 hands-free conversations (35.5\%) and of 23 hand-held conversations (18.5\%). Visual-manual interactions with the phone were observed in 57 phone use cases $(46.0 \%)$.

Overall, in the sample there were 238 passenger cars $(96.0 \%)$ and 10 minivans $(4.0 \%) .108$ observed drivers were women (43.5\%) and 140 were men (56.5\%). Target group and control group had the same gender distribution. Of the 54 female phone users 27 (50\%) made a visual-manual interaction, and the 70 male phone users were calling in 40 cases $(57.1 \%)$ and interacting visual-manually in 30 cases $(42.9 \%)$. The chi-squared revealed no significant relationship between gender and phone use mode, $X^{2}(2, N=124)=0,626$, n.s. .

131 drivers $(52.8 \%)$ were estimated to be between thirty and fifty years of age, 86 drivers (34.7\%) were judged as being over fifty and 31 drivers $(12.5 \%)$ appeared to be under thirty. The distribution of estimated age did not significantly differ between the control and target group, $X^{2}(2, N=248)=4,727$, n.s. (table 1), neither did it between users who were calling and those who were using the phone in a visual-manual interaction, $X^{2}(2, N=124)=1,893$, n.s. (table 2).

Table 1: Cross tabulation of phone use and estimated age: frequency counts and contingent percentages.

\begin{tabular}{lrcrrr}
\hline & $<\mathbf{3 0}$ & & $\mathbf{3 0 - 5 0}$ & & \multicolumn{2}{c}{$\sum$} \\
\hline Non-user & & 58 & 51 & $\mathbf{1 2 4}$ \\
\multirow{2}{*}{ User } & 15 & $46.8 \%$ & $41.1 \%$ & $100 \%$ \\
& 16 & 73 & 35 & $\mathbf{1 2 4}$ \\
\hline$\sum$ & $12.9 \%$ & $58.9 \%$ & $28.2 \%$ & $100 \%$ \\
\hline & $\mathbf{4 4}$ & $\mathbf{2 3}$ & $\mathbf{5 7}$ & $\mathbf{2 4 8}$ \\
\hline
\end{tabular}


21

5

Table 2: Cross tabulation of phone use mode and estimated age: frequency counts and contingent percentages.

\begin{tabular}{lrrrrr}
\hline & $<\mathbf{3 0}$ & $\mathbf{3 0 - 5 0}$ & & \multicolumn{5}{c}{$\sum$} & \\
\hline call & & & 38 & 22 & $\mathbf{6 7}$ \\
& 7 & $56.7 \%$ & $32.8 \%$ & $100 \%$ \\
visual-manual & $10.5 \%$ & 35 & 13 & $\mathbf{5 7}$ \\
interaction & 9 & $61.4 \%$ & $22.8 \%$ & $100 \%$ \\
\hline$\sum$ & $15.8 \%$ & $\mathbf{7 3}$ & $\mathbf{3 5}$ & $\mathbf{1 2 4}$ \\
\hline
\end{tabular}

\subsection{Existence of phone use strategy}

The first research question was addressed by analysing the distribution of the phone use modes that were observed in the red traffic light situation, and by distinguishing between uses that were already ongoing when the drivers entered the observation zone and those that were initiated during the waiting time at the traffic light. About half of the observed phone uses were initiated while waiting at the red traffic light and the other half were already ongoing when the driver arrived at the red traffic light. However, as illustrated in table 3 and confirmed by the chi-squared test, visual-manual interactions had significantly higher chances to be initiated at the red traffic light, whereas calls were more likely to be already ongoing, $X^{2}$ $(1, N=124)=68,144, p=.000, \mathrm{phi}=.74$. Among the 58 calls that were already ongoing, 42 were hands-free conversations and 16 hand-held conversations. Two hands-free conversations and seven hand-held conversations were initiated.

Table 3: Cross tabulation of phone use mode and beginning of phone use in relation to red traffic light situation: frequency counts and contingent percentages.

\begin{tabular}{|c|c|c|c|}
\hline & ongoing & initiated & $\sum$ \\
\hline \multirow[t]{2}{*}{ call } & 58 & 9 & 67 \\
\hline & $86.6 \%$ & $13.4 \%$ & $100 \%$ \\
\hline visual-manual & 7 & 50 & 57 \\
\hline interaction & $12.3 \%$ & $87.7 \%$ & $100 \%$ \\
\hline$\sum$ & 65 & 59 & 124 \\
\hline
\end{tabular}




\subsection{Effectiveness of phone use strategy}

2 In order to determine whether the phone use was restricted to the red traffic light situation, the

3 phone use observations were analysed regarding their interruption once the traffic light turns

4 green. Table 4 shows the distribution of stopped and continued phone uses. Overall, the 5 majority of phone uses extended beyond the red traffic light phase. Still, the phone use

6 behaviour differs significantly between calls and visual-manual interactions. Statistical testing

7 reveals that visual-manual interactions tend to be stopped before the vehicle moves off, while

8 calls tend to be continued, $X^{2}(1, N=124)=44.358, p=.000$, phi $=.60$. The seven interrupted calls

9 that were observed were exclusively hand-held conversations.

Table 4: Cross tabulation of phone use mode and interruption of phone use after end of red traffic light situation: frequency counts and contingent percentages.

\begin{tabular}{|c|c|c|c|}
\hline & continued & interrupted & $\sum$ \\
\hline \multirow[t]{2}{*}{ call } & 60 & 7 & 67 \\
\hline & $89.6 \%$ & $10.4 \%$ & $100 \%$ \\
\hline visual-manual & 18 & 39 & 57 \\
\hline interaction & $31.6 \%$ & $68.4 \%$ & $100 \%$ \\
\hline$\sum$ & 78 & 46 & 124 \\
\hline
\end{tabular}

The indicator of impaired situation awareness, delayed start when the traffic light turns green, was observed more frequently for users than non-users (table 5). The chi-squared test confirms the relationship between delayed start and phone use, $X^{2}(1, N=248)=9.185, p=.002$, phi=.19. Among phone users, visual-manual interactions are more likely to be associated with a delayed start than calls, as illustrated in table $6, X^{2}(1, N=124)=16.729, p=.000, \mathrm{phi}=.37$. However, no significant effect was detected when examining the relationship between interrupted and continued phone use and delayed start at the green traffic light, $X^{2}(1$, $N=124)=0.266$, n.s. $($ table 7$)$. 


\begin{tabular}{lrrr}
\hline & no delayed start & delayed start & $\sum$ \\
\hline non-user & 116 & 8 & $\mathbf{1 2 4}$ \\
& $93.5 \%$ & $6.5 \%$ & $100 \%$ \\
user & 100 & 24 & $\mathbf{1 2 4}$ \\
& $80.6 \%$ & $19.4 \%$ & $100 \%$ \\
\hline$\sum$ & $\mathbf{2 1 6}$ & $\mathbf{3 2}$ & $\mathbf{2 4 8}$ \\
\hline
\end{tabular}

3

4 Table 6: Cross tabulation of phone use mode and delayed start when traffic light turns green: frequency counts

5 and contingent percentages.

6

\begin{tabular}{lrrr}
\hline & no delayed start & delayed start & $\sum$ \\
\hline call & 63 & 4 & $\mathbf{6 7}$ \\
& $94.0 \%$ & $6.0 \%$ & $100 \%$ \\
visual-manual & 37 & 20 & $\mathbf{5 7}$ \\
interaction & $64.9 \%$ & $35.1 \%$ & $100 \%$ \\
\hline$\sum$ & $\mathbf{1 0 0}$ & $\mathbf{2 4}$ & $\mathbf{1 2 4}$ \\
\hline
\end{tabular}

7

8

contingent percentages.

Table 7: Cross tabulation of interruption of phone use and delayed start when traffic light turns green: frequency counts and contingent percentages.

\begin{tabular}{lrrr}
\hline & no delayed start & delayed start & $\sum$ \\
\hline interrupted & 36 & 10 & $\mathbf{4 6}$ \\
& $78.3 \%$ & $21.7 \%$ & $100 \%$ \\
continued & 64 & 14 & $\mathbf{7 8}$ \\
& $82.1 \%$ & $17.9 \%$ & $100 \%$ \\
\hline$\sum$ & $\mathbf{1 0 0}$ & $\mathbf{2 4}$ & $\mathbf{1 2 4}$ \\
\hline
\end{tabular}

\section{Discussion}

The data collected in this study gives insights into the patterns of phone use at urban intersections. Although hand-held phone use and visual-manual interactions are not allowed behind the wheel in France, not even while stopped in traffic, these phone use modes represent more than half of the phone uses observed at the red traffic light. Gender and age were comparable among users and non-users in this study, contrary to previous findings suggesting that younger age and being male was a predictor for in-car phone use (Pöysti et al., 2005; Brusque \& Alauzet, 2008; Sullman, 2012). The distributions of phone use modes were not related to gender or age either. 
1 In response to the first research question, a large effect was found when comparing the initiation of phone use at the red light between calls and visual-manual interactions. The results of this study show that drivers take the chance to initiate visual-manual interactions with the phone while waiting at the red traffic light. On one hand, the waiting time might incite them to interact with their phone, possibly out of boredom or due to the reflex to check the phone as soon as the opportunity arises. On the other hand, drivers might be aware of the interference visual-manual interactions with the phone represent and wait for a driving situation with low demands before engaging in this mode of phone use. The majority of visual-manual interactions do not require immediacy and can be strategically delayed, e.g. a driver can wait before answering a text message or before checking email. This is not necessarily the case for phone calls, which were more likely to be ongoing when the drivers arrived at the red traffic light in the present study. Firstly, the drivers might answer incoming calls immediately instead of calling back when the situation is more appropriate and secondly, the call might have started in a low demand situation (e.g., another traffic light) and last longer. The legality of hands-free phone use while driving could also play a role in this context. Avoiding fines could be a motivation to restrict phone use and the fact that handsfree phoning is allowed might even encourage drivers to consider that it does not increase crash risk (White et al., 2010) and, hence, that there is no need to adapt their phone use behaviour.

Correspondingly, a large effect was found between calls and visual-manual interactions concerning the second research question. The results on the cessation of phone use when the traffic light turns green confirm that calls are more likely to be prolonged beyond the stopping situation than visual-manual interactions. This finding reflects the incompatibility of visualmanual interactions with the driving task within intersections due to their higher distractive potential. However, it cannot be determined whether this phone use strategy is the result of a 
1 conscious decision based on the drivers' perception of crash risk or whether it is only the

2 consequence of the difficulties to comfortably deal with the concurrent tasks.

3 At the same time, the observation of a majority of phone uses extending beyond the red traffic

4 light phase represents a potential safety concern, given that calls provoke cognitive distraction

5 (e.g., Strayer \& Drews, 2007). This distraction has been found to narrow the perceptual field

6 of view (Recarte \& Nuñes, 2000), which is especially relevant for the driving performance in

7 complex environments. The phone use behaviour observed in the present study concurs with

8 previous findings on drivers' underestimation of cognitive distraction by a conversation and

9 their judgement of visual-manual distractions as hazardous (McEvoy et al., 2005; Huth \& Brusque, 2013). The results confirm that drivers particularly regulate their exposure to phone use when it includes visual-manual distraction, as concluded from a literature review on drivers' adaptation to mobile phone use while driving (Huth \& Brusque, 2013).

The results on the delayed start when the traffic light turns green support the claim that mobile phone use affects situation awareness. A small effect was found when comparing users and non-users, while the comparison of phone use mode and delayed start revealed a medium size effect. As it was expected, more demanding phone use (visual-manual interactions) is more often associated with impaired situation awareness. Interestingly, no distinction could be made between the effects of interrupted and persisting phone use. Continued phone use was not associated to significantly more delayed starts than phone use that was interrupted. These data suggest that the distractive effects of phone use might last moment at which the traffic light turns green might be too close in time as to permit an 
1 In conclusion, choosing red traffic light situations to interact with the phone does not seem to

2 interfere with the driving activity at a first glance, but the results show that drivers are limited in their ability to monitor the situation and this impaired situation awareness implies slower reactions to changing traffic situations. The threat of intersection scenarios lies in the contrast of a situation with very low demands while waiting at red traffic light and a highly complex situation when moving off, which requiring manoeuvres, interactions with other road users, gap estimations, etc. Phone interactions might not be flexible enough to adapt to the abrupt change of from one situation to the other. The findings of the present study suggest that red traffic light situations are too short as to allow effective application of phone use strategies. This holds especially for calls, given that they tend to be longer and less easy to interrupt than visual-manual interactions. As a result, phone use extends beyond stopping situations and might lead to unintended concurrence of two tasks whose added demands exceed the maximum information processing capacity of the driver. Visual-manual distraction especially represent a hazard since they capture an essential share of the drivers' attentional resources, while the risk related to calls is constituted by a longer exposure to an underestimated cognitive distraction. At the same time, inner-urban traffic is characterized by relatively low speed and may often be part of a driver's familiar environment, which may induce drivers to underestimate the associated accident risk. The findings on lack of situation awareness hint towards a possible increase in crash risk due to driver distraction by phone use at traffic lights. The types of crashes that could occur in this context range from low severity crashes, such as rear-end collisions that could be provoked by the following vehicle when a distracted driver does not move off at the green traffic light, to crashes with potentially severe outcome, such as those involving pedestrians or front-side collisions after red light running by a distracted driver or failure to give way at left turns with oncoming traffic during the green phase of the traffic light. 
1 The limitations of this study are mostly inherent to the methodology applied. Its main

2 advantage is the direct observation of behaviour in its natural environment. While relying on

3 human observers provides certain flexibility during data collection and allows registering

4 inferential variables that are based on expert judgement, some drawbacks have to be taken

5 into account. First, manual data collection by the observers cannot be expected to be

6 completely exhaustive in this study. The capacities of the observers could be limited due to

7 impaired visibility to vehicles in the queue that were farther away from the observer and to

8 vehicles on the far-side lane that were obstructed by vehicles on the near-side lane. Moreover,

9 phone users could be missed when the observers were busy coding other drivers. Besides,

10 determining phone use rates does not make sense in the scope of this study, and the total

11 number of vehicles in the observation zone was not counted to that end.

Second, generalizability might be limited due to the relatively small sample. However, efforts were made to randomize as much of the design as possible so as to increase generalizability. Three sites were included into the study and the three observation sessions at each site were conducted at different days of the week and at three different times (between 4 and $7 \mathrm{pm}$ ). Still, it would be interesting to replicate this study on other French sites and in other countries.

Furthermore, the observers switched roles during data collection, registering the target group and the control group alternately. Yet, the use of inferential variables could affect the quality of the collected data. For increased reliability and validity of data collection, the researchers reached a high degree of familiarity with the contents of the observation during the ad hoc observations and the observer training. Moreover, the red traffic light situation represented a static traffic situation that was long enough for the observer to adequately assess the situation. Lastly, the standardized observation grid and a strict observation protocol were implemented in order to minimize possible observer bias. 
1 Nevertheless, the taxonomy of phone uses remains relatively unspecific. While hands-free and

2 hand-held calls as well as visual-manual interactions could be distinguished, the exact phone activity could not be determined. For instance, no assumption can be made on the relatedness of the phone use to the driving activity. Visual-manual interactions could represent the use of mobile applications, such as navigation, parking information or other types of driving assistance, whereas calls could be made to receive directions or to inform about late arrival. These issues need to be dealt with in self-report studies, and it should be tested in a controlled environment how different phone use content affects attention. The distinction between calls and visual-manual interactions in the present study is solely based on the underlying distraction mechanism, i.e. whether visual distraction is included or not.

Finally, the presence of observers could change the behaviour of drivers. In order to counteract this bias, the observers used inconspicuous clothing and were placed on the sidewalk of the opposite lane. In addition, the observation zone only comprised the first four vehicles at the traffic light, which gave the drivers less time to detect the observers.

Further research is needed so as to better understand the factors that underlie drivers' decisions to use the phone in different driving situations and to identify the factors that could prevent phone use while driving. For example, it should be analysed if phone use that is not compatible with the driving situation is due to a judgement error, or if it is the neglect of a known risk due to the priority of a competing need. Phone use behaviour is often driven by internal motives concerning the management of time and resources, the enjoyment of technology use, socializing and general lifestyle attitudes (Lerner, 2005; White et al., 2010). Moreover, a differentiation between active decisions or impulse-driven habits would help defining appropriate countermeasures. This aspect is particularly relevant since smartphones start shaping the behaviour of many people in a way that is not always socially appropriate but might be driven by an addiction-like urge to stay connected and to stay informed (Chóliz, 
1 2012). In this context, an analysis of the social component of phone use while driving is also

2 of interest. Heavy mobile phone use from an early age is common in today's society, phone

3 use can take place anywhere and at anytime, and distracted driving has become a normative

4 behaviour. In a recent study, drivers who were confronted with crash scenarios recognized the

5 greater responsibility of a distracted driver for an accident, but they did not suggest greater

6 punishments (fines and jail time) for this driver than for an undistracted driver (Atchley et al.,

7 2012). The social pressure to communicate in combination with the missing social reprobation

8 of mobile phone use while driving could incite drivers to engage in this distracting activity. It

9 could therefore be beneficial to change social norms regarding mobile phone use in traffic, 10 such as it has been achieved regarding drink driving (Atchley et al., 2012).

11 Further countermeasures could be based on technical solutions that block the phone or limit its functions when the car is moving. However, their technical feasibility and user acceptance need to be assessed. Alternatively, more driving task compatible human-machine interfaces which shift visual-manual behaviour to a speech user interface could help to better manage distraction and mitigate the effects of intersection distraction. A message system interface giving the driver control over the timing of speech-based e-mail presentation, e.g., proved to have a positive effect on driving performance under most circumstances, whereas it was detrimental in highly demanding situations (Jamson et al., 2004). The cognitive costs associated with the decision-making process imposed by the system use need to be considered.

Technical solutions based on a real-time diagnosis of the driving situation have been designed to assist the driver in deciding when to carry out secondary tasks. Depending on the situational demands, these so-called workload managers regulate the information input that is potentially distractive, for instance by diverting an incoming call automatically to the mailbox if the driver is performing a manoeuvre at an intersection, and give advice to the driver when 
1 not to perform a self-paced distractive task, e.g. outgoing calls. Simulator tests on such a

2 system revealed that drivers positively rate the support in combining the secondary task with

3 driving, but that they often overrule the system messages (Mühlbacher et al., 2010). It has to

4 be carefully evaluated whether such measures can resolve the issue of phone use that extends

5 over changing driving situations and if they are adapted to intersections, where contrasting

6 situational demands alternate with one another.

\section{Acknowledgements}

9 The research leading to these results has received funding from the European Community's Seventh Framework Programme (FP7/2007-2013) under grant agreement no. 238833/ADAPTATION project (www.adaptation-itn.eu).

\section{References}

Bayer, J.B. \& Campbell, S.W., 2012. Texting while driving on automatic: Considering the frequency-independent side of habit. Computers in Human Behavior, 28, 2083-2090.

Brace C.L., Young K.L., Regan M.A. Analysis of the literature: the use of mobile phones while driving. Monash University Accident Research Centre, 2007 \& Vagverket, Swedish Road Administration, 2007.

Brusque, C. \& Alauzet, A. (2008). Analysis of the individual factors affecting mobile phone use while driving in France: Socio-demographic characteristics, car and phone use in professional and private contexts. Accident Analysis and Prevention, 40, 35-44. 
1 Bruyas M.P. (2013). Impact of mobile phone use on driving performance: review of experimental literature. In Regan M.A., Lee J.D., Victor T.W. (eds.): Driver Distraction and Inattention. Advances in Research and Countermeasures, Volume 1. UK: Ashgate, pp. 2934309.

5 Charlton, S. G. (2009). Driving while conversing: Cell phones that distract and passengers 6 who react. Accident Analysis \& Prevention, 41(1), 160-173.

7 Chóliz, M. (2012). Mobile-phone addiction in adolescence: The Test of Mobile Phone Dependence (TMD). Progress in Health Sciences, Vol. 2, 33-44.

9 Cooper, P. J., Zheng, Y., Richard, C., Vavrik, J., Heinrichs, B., \& Siegmund, G. (2003). The impact of hands-free message reception/response on driving task performance. Accident Analysis \& Prevention, 35(1), 23-35.

Drews, F.A., Yazdani, H., Godfrey, C.N., Cooper, J.M. \& Strayer D.L. (2009). Text messaging during simulated driving. Human Factors, 51(5), 762-70.

Eby, D.W. (2011). Naturalistic Observational Field Techniques for Traffic Psychology Research. . In B.E. Porter (Ed). Handbook of Traffic Psychology. Academic Press, London, pp. 61-72.

Endsley, M.R. (1988). Design and evaluation for situational awareness enhancement. Proceedings of the Human Factors Society 32nd Annual Meeting. HFES, Santa Monica, CA, SAGE, pp. 97-101. Habibovic, A. \& Davidsson, J. (2012). Causation mechanisms in car-to-vulnerable road user 
1 Hickman, J.S, Hanowski, R.J. \& Bocanegra, J. (2010). Distraction in commercial trucks and

2 buses: assessing the prevalence and risk in conjunction with crashes and near-crashes. US

3 Department of Transportation, Federal Motor Carrier Safety Administration, Report, 4 September 2010.

5 Horrey, W.J. \& Wickens, C.D. (2007). In-vehicle glance duration: Distributions, tails and 6 model of crash risk. Transportation Research Record 2018, 22-28.

7 Hosking, S. G., Young, K. L. \& Regan, M.A. (2009). The effects of text messaging on young 8 drivers. Human Factors 51(4), 582-92.

9 Huth, V. \& Brusque, C. (2013). Drivers' adaptation to mobile phone use: interaction strategies, consequences on driving behaviour and potential impact on road safety. In A. Stevens, C. Brusque \& J. Krems (Eds). Driver adaptation to information and assistance systems. IET published book, pp. 173-196.

International Telecommunication Union [ITU] (2014). Mobile-cellular telephony [online]. Available from http://www.itu.int/ITU-D/ict/statistics/ [Accessed 18 Mar 2014]. Jamson, A., Westermann, S., Hockey, G. \& Carsten, O. (2004). Speech-based e-mail and driver behaviour: Effect of an in-vehicle message system interface. Human Factors: The Journal of the Human Factors and Ergonomics Society, 46, 625-39.

Kircher, K., Patten, C. \& Ahlström, C. (2011). Mobile telephones and other communication devices and their impact on traffic safety. VTI Report 729A.

Klauer, S.G., Dingus, T.A., Neale, V.L., Sudweeks, J.D., Ramsey, D.J. (2006). The Impact of Driver Inattention on Near-Crash/Crash Risk: An Analysis Using the 100-Car Naturalistic Driving Study Data. DOT HS 810 594, Virginia Tech Transportation Institute. 
1 Lerner, N. \& Boyd, S. (2005). On-road study of willingness to engage in distracting tasks.

2 DOT HS-809-863, National Highway Traffic Safety Administration, Washington DC.

3 McCartt, A.T., Hellinga, L.A. \& Braitman, K.A. (2006). Cell phones and driving: review of 4 research. Traffic Injury Prevention 7, 89-106.

5 McEvoy, S.P., Stevenson, M.R., McCartt, A.T., Woodward, M., Haworth, C., Palamara, P. \&

6 Cercarelli, R. (2005). Role of mobile phones in motor vehicle crashes resulting in hospital 7 attendance: a case-crossover study. British Medical Journal 331, 428-30.

8 McKnight, J.A. \& McKnight, S.A. (1993). The effect of cellular phone use upon driver 9 attention. Accident Analysis \& Prevention 25, 259-65.

Mühlbacher, D., Totzke, I., Schömig, N. \& Rothe, S. (2010). Workload management while driving: Do subjects' judgements reflect driving safety? Proceedings of the European Conference on Human Centred Design for ITS. Berlin, Germany. Lyon: HUMANIST, pp.289-99.

Observatoire National Interministériel de la Sécurité Routière [ONISR] (2013). La sécurité routière en France - Bilan de l'accidentalité de l'année 2012. Paris, France.

OECD/ITF (2014). Road Safety Annual Report 2014. Paris, France.

Olson, R., Hanowski, R., Hickman, J. \& Bocanegra, J. (2009). Driver distraction in commercial vehicle operations. US Department of Transportation, Federal Motor Carrier Safety Administration: 49.

Pickrell, T.M. \& Ye, T.J. (2010). Driver Electronic Device Use Observation Protocol. Traffic Safety Facts Research Note, US Department of Transportation, Highway Safety Administration, Report No. DOT HS 811361. 
1 Pöysti, L., Rajalin, S. \& Summala, H. (2005). Factors influencing the use of cellular (mobile)

2 phone during driving and hazards while using it. Accident Analysis and Prevention, 37, 47-51.

3 Rauch, N., Gradenegger, B. \& Krüger, H.-P. (2008). User strategies for the interaction with 4 in-vehicle devices while driving'. IET Intelligent Transport Systems, 2(4), 266-75.

5 Recarte, M.A. \&Nuñes, L.M. (2000). Effects of verbal and spatial-imagery tasks on eye 6 fixation while driving. Journal of Experimental Psychology: Applied, 6(1), 31-43.

7 Redelmeier, D.A., Tibshirani, R.J. (1997). Association between cellular telephone calls and 8 motor vehicle collisions. The New England Journal of Medicine, 336(7), 453-58.

9 Sandin, J. (2009). An analysis of common patterns in aggregated causation charts from 10 intersection crashes. Accident Analysis \& Prevention, 41(3), 624-632.

Schömig, N., Metz, B. \& Krüger, H.-P. (2011). Anticipatory and control processes in the interaction with secondary tasks while driving. Transportation Research Part F: Traffic Psychology and Behaviour, 14(6), 525-38.

Strayer, D. L. \& Drews, F. A. (2007). Multi-tasking in the automobile. In A.F. Kramer, D.A.

Wiegmann \& A. Kirlik (Eds.). Applied attention: From theory to practice, Oxford University Press, pp. 121-133.

Strayer, D. L., Drews, F. A. \& Johnston, W. A. (2003). Cell phone-induced failures of visual attention during simulated driving. Journal of experimental psychology: Applied, 9(1), 23-32. Driver's exposure to distractions in their natural driving environment. Accident Analysis \& Prevention, 37(6), 1093-1101. 
1 Sullman, M.J. (2012). An observational study of driver distraction in England. Transportation

2 research part F: traffic psychology and behaviour, 15(3), 272-278.

3 Summala, H. (1997). Hierarchical model of behavioral adaptation and traffic accidents. In T.

4 Rothengatter \& E. Carbonell Vaya (eds.): Traffic and Transport Psychology. Amsterdam:

$5 \quad$ Pergamon Press, pp. 41-52.

6 Walker, L., Williams, J. \& Jamrozik, K. (2006). Unsafe driving behaviour and four wheel

7 drive vehicles: observational study. bmj, 333(7558), 71-74.

8 White, K., Hyde, M., Walsh, S. \& Watson, B. (2010). Mobile phone use while driving: An 9 investigation of the benefits influencing drivers' hands-free and hand-held mobile phone use. Transportation Research Part F: Traffic Psychology and Behaviour, 13(1), 9-20.

11 World Health Organization [WHO] (2011). Mobile phone use: a growing problem of driver distraction. Geneva, Switzerland.

13 Young, K.L., \& Salmon, P.M. (2012). Examining the relationship between driver distraction 14 and driving errors: A discussion of theory, studies and methods. Safety Science, 50(2), 165174.

16 Young, K.L., Rudin-Brown, C.M., \& Lenné, M.G. (2010). Look who’s talking! A roadside survey of drivers' cell phone use. Traffic Injury Prevention, 11, 555-560. 